diagnosis and management of obstetric brachial plexus injury. (Int Pediatr 1995;10:208-213). Prenatal non-obstetric trauma produces a fixed anatomical deformity and EMG fibrillations present at or soon after birth. These authors recommend early EMG only in the exceptional cases without history of a difficult birth, those infants with a low birth weight, and with signs of congenital muscular atrophy or contractures indicative of prenatal pathology. Congenital chicken pox, amniotic bands, and pseudoparalysis due to fractures or osteomyelitis may mimic Erb's palsy. I have seen cases of congenital syphilitic osteochondritis that presented as pseudoparalysis and were treated initially as an obstetric Erb's palsy.

Caution is required in the interpretation of fibrillation potentials in the newborn infant. Transient spontaneous potentials, similar in amplitude, duration, and frequency to fibrillation potentials, have been reported in "essentially normal" premature and full-term infants. Jones HR, Bolton CF, Harper CM (Pediatric Clinical Electromyography, Philadelphia, LippincottRaven, 1996) have reviewed in detail the electromyography of newborns and reasons for inconclusive or erroneous EMG clinical correlations. Animal data suggest that fibrillation potentials may occur within 2 days after nerve section. EMG must be performed during the first 12 to 48 hours of life in order to attempt a distinction between prenatal and perinatal nerve injury. Further studies are needed to determine more accurately the temporal relationship of injury to onset of denervation potentials in the newborn. It might be imprudent to rely on EMG findings alone to assign cause of brachial plexus injury in obstetric litigation cases.

\title{
RADIAL MONONEUROPATHIES
}

Sixteen cases of radial mononeuropathy, presenting with wristdrop and seen in the EMG laboratory at Children's Hospital, Boston, during 16 years, 1979-95, were analysed. Eight were atraumatic, including 2 in newborns, related to compression in 6 and entrapment in 2. Eight were caused by fractures or lacerations. The lesions localized by EMG were in the distal main radial nerve trunk in $9(56 \%)$, the posterior interosseous nerve in $5(31 \%)$, and in the proximal main radial nerve trunk in $2(13 \%)$. Demyelinating lesions in 4 cases improved within 6-12 weeks, and axonal injuries in 13 took between 6 weeks and 18 months to improve or recover. Two showed no improvement in 4 years, and 1 caused by sclerotherapy for a nevus was progressive. (Escolar DM, Jones HR Jr, Pediatric radial mononeuropathies: a clinical and electromyographic study of sixteen children with review of the literature. Muscle Nerve July 1996;19:876-883). (Reprints: H Royden Jones Jr MD, Department of Neurology, Lahey Hitchock Medical Center, 41 Mall Road, Burlington, MA 01805).

COMMENT. These authors have previously determined that an EMG taken within 1-2 days of birth and showing fibrillation potentials is required to support a diagnosis of intrauterine prenatal-onset neuropathy. (Jones HR et al. Intrauterine onset of a mononeuropathy: peroneal neuropathy in a newborn with EMG findings compatible with a prenatal onset. Muscle Nerve 1996;19:8891). The precise temporal profile for signs of denervation in the neonate with birth injury has not been well defined, and an EMG performed later than 48 hours after birth may not distinguish prenatal from perinatal injuries. The distribution of the EMG abnormalities differentiates the radial neuropathies from the brachial plexus palsies. 